\title{
Effiziente Schutzlack-Härtung mit Infrarot
}

$\mathrm{O}$ Korrosionsschutz für Nähte von Blechdosen, Steinschlagschutzbeschichtung für Autos oder AntiFingerprint-Lack für Edelstahloberflächen: Alle diese funktionalen Beschichtungen können durch Infrarot-Systeme schnell und effizient getrocknet werden, ohne dabei ihre speziellen Eigenschaften zu verlieren. Der geringe Platzbedarf und der gezielte Energieeinsatz machen Infrarot zu einer attraktiven Wärmequelle. Heraeus Noblelight präsentiert in der Sonderschau Praxispark im Rahmen der Hannover Messe anwendungsoptimierte Infrarot-Strahler und -Systeme. Lacksysteme mit funktionalen Eigenschaften, viele davon Nanolacke, müssen sehr homogen aufgetragen und dann schnell getrocknet werden. InfrarotStrahler übertragen Wärme kontaktfrei und effizient. Dadurch wird das Anhaften von störenden Partikeln im empfindlichen Lack während der Trocknung minimiert. Darüber hinaus besitzt Infrarot-Strahlung eine vielfache Wärmeübertragungskapazität im Vergleich zu Konvektion.
Federn aus Stahl oder Nahtstellen an Blechdosen werden während der Fertigung mit wasserbasierendem Schutzlack gegen Korrosion beschichtet. Der Wasserlack muss zuverlässig trocknen, dabei soll die Metallmasse jedoch nicht komplett aufgeheizt werden. Wasser verdunstet durch eine Bestrahlung mit mittelwelligen Infrarot-Strahlern besonders schnell. Die meisten Lacke absorbieren Infrarot-Strahlen sehr gut aufgrund ihrer chemischen Zusammensetzung. Dabei dringen die Strahlen teilweise in die Lackschicht ein und sorgen damit für ein rasches Verdunsten der Lösungsmittel. Da Wasser wesentlich mehr Energie zum Verdunsten benötigt als organische Lösungsmittel, ergibt sich in der Praxis
Handlungsbedarf für bestehende Anlagen. Eine Verlängerung bestehender Trockner scheitert oft aus Platzgründen. Hier hilft ein sogenannter IR-Booster, eine vor dem Ofen oder in den Einlauf des Ofens befindliche IR-Erwärmungsstation. Sie sorgt für einen raschen Temperaturanstieg auf die Trocknungstemperatur und ermöglicht die Einhaltung der erforderlichen Verweilzeit.

Tel. 06181 35-8492,

www.heraeus-noblelight.com

Halle 3, Stand E 14

\section{Leistungsstarke Filter für Galvanobäder}

$\mathrm{B}^{\mathrm{s}}$ ohncke stellt auf der Surface Technology Standardprodukte und Neuheiten aus, die zur Standzeitver-

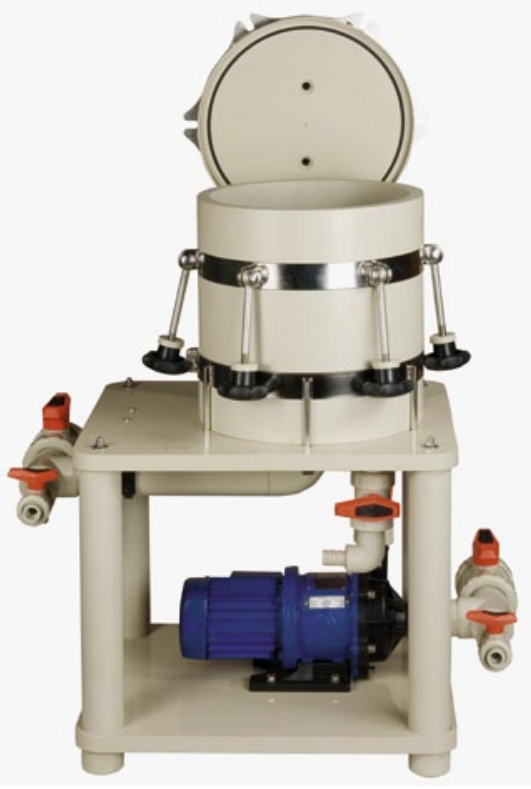

längerung der Bäder, Sicherheit und Verfügbarkeit der Galvanikanlagen sowie zur Senkung der Filtrationskosten beitragen. Mit den Badinhalten werden auch die Umwälzraten in den Galvanobädern werden immer größer. Entsprechend steigen die Ansprüche an die Filtertechnik, die mit den weiterentwickelten Filtergeräten der Serien 180, 250, 355 und 575 abgedeckt werden. Die Filterleistung kann bis 50 $\mathrm{m} 3 / \mathrm{h}$ betragen. Große Filterflächen ermöglichen lange Standzeiten und große Schmutzfrachten. Alle üblichen Filterarten, wie Kerzen, Papier, Kunststoff Anschwemmelemente und Tuch können ohne Umbau eingesetzt werden. Damit soll gewährleistet werden, dass stets die geeigneten beziehungsweise kostengünstigsten Filtermittel eingesetzt werden können.

Tel. 06126 9384-0, www.bohncke.de

Halle 3, Stand D 54(3)

\section{Galvanikanlagen für dekorative und funktionelle Oberflächen}

Maler Lemmen stellt auf dem Gemeinschaftsstand "Welt der Oberflächen" neben kompakten Kleingalvanikanlagen und Wannensystemen für funktionelle und dekorative Oberflächenbehandlung, Galvanikanlagen zum elektrochemischen Polieren und Engraten, Anodisieren von Titan, Eloxieren von Aluminium, Beizen, Vorreinigen und Passivieren von Werkstücken aus. Die Anlagen und Wannensysteme sind universell für die Vorbehandlung, Veredelung und Nachbehandlung von Oberflächen der Leiterplatten- und Schmuckindustrie, Medizintechnikund industriellen Kleinteilefertigung einsetzbar. Der Anlagenaufbau beinhaltet alle erforderlichen Anlagenkomponenten zur Realisierung von qualitativ hochwertigen.

Tel. 09342 7851, www.walterlemmen.de

Halle 3, D 54(7) 